\title{
TIPIFICAÇÃO DE SINTOMAS RELACIONADOS \\ À VOZ E SUA PRODUÇÃO EM PROFESSORES \\ IDENTIFICADOS COM AUSÊNCIA DE ALTERAÇÃO VOCAL \\ NA AVALIAÇÃO FONOAUDIOLÓGICA
}

\author{
Typification of symptoms related to voice and its production \\ in teachers identified with absence of vocal alteration \\ in Speech-Language Pathology evaluation
}

Emilse Aparecida Merlin Servilha ${ }^{(1)}$, Joyce Pena ${ }^{(2)}$

\begin{abstract}
RESUMO
Objetivo: tipificar os sintomas relacionados à voz e sua produção auto referidos por professoras, cujas vozes foram identificadas como saudáveis na avaliação fonoaudiológica. Métodos: participaram 36 docentes, idade média de 37 anos, solteiras (75\%) e escolaridade superior $(83,33 \%)$ da rede municipal de ensino de uma cidade do interior do estado de São Paulo. Os docentes responderam a um questionário e referiram a alteração, suas vozes foram gravadas e submetidas à avaliação fonoaudiológica perceptivo-auditiva e posteriormente comparados os dois tipos de avaliação. Procedeu-se a caracterização sócio demográfica, condições ambientais e organizacionais do trabalho dos docentes e seus sintomas tipificados e submetidos à análise estatística descritiva. Resultados: constatou-se a presença de poeira $(91,67 \%)$, ruído $(75 \%)$ e excesso de trabalho $(88,88 \%)$, falta de tempo para desenvolver as atividades na escola $(88,88 \%)$ e fiscalização constante do desempenho $(33,33 \%)$. Quanto à voz, a alteração foi percebida há mais de quatro anos $(30,56 \%)$, secundária ao seu uso intensivo $(94,44 \%)$ e ao estresse $(61,11 \%)$, classificada como moderada $(66,67 \%)$, sendo proeminentes como sintomas auditivos, a rouquidão e o cansaço ao falar, ambos com $69,44 \%$ e como sintomas proprioceptivos, o pigarro $(63,88 \%)$ e a garganta seca $(61,11 \%)$. A comparação entre os sintomas mostra que os proprioceptivos $(63,26 \%)$ foram mais mencionados do que os auditivos $(36,73 \%)$. Conclusão: os professores trabalham em ambientes adversos à saúde e à voz; a prevalência de sintomas proprioceptivos foi maior que os auditivos, o que pode ter interferido na avaliação perceptual de suas vozes pelas fonoaudiólogas que contaram apenas com a pista auditiva.
\end{abstract}

DESCRITORES: Saúde do Trabalhador; Voz; Distúrbios da Voz; Docentes; Qualidade da Voz

\section{INTRODUÇÃO}

A voz é fator relevante para a atuação do professor em sala de aula, como recurso didático e de

(1) Fonoaudióloga; Professora da Faculdade de Fonoaudiologia da Pontifícia Universidade Católica de Campinas, PUCCAMP, Campinas, SP, Brasil; Doutora em Ciências pela Pontifícia Universidade Católica de Campinas.

(2) Aluna do Curso de Graduação em Fonoaudiologia da Pontifícia Universidade Católica de Campinas, PUCCAMP, Campinas, SP, Brasil.

Conflito de interesses: inexistente interação professor-aluno ${ }^{1,2}$, por isso, deve ser preservada de agentes agressivos e mantida a integridade de suas qualidades como um aspecto importante da saúde geral e qualidade de vida ${ }^{3}$.

No contexto pedagógico, as condições do ambiente de trabalho (acústica, nível de competição sonora ambiental, umidade, poeira), características da atividade (extensão da jornada de trabalho, tempo de exposição oral durante as aulas, manutenção de vários empregos) e falta de preparo/treinamento para o uso adequado da voz são fatores que podem contribuir para o abuso vocal, gerando alterações vocais em diferentes níveis de 
frequência e severidade ${ }^{4,5}$, afastamento temporário ou permanente da sala de aula ${ }^{6}$.

Os estudos sobre as relações entre saúde e trabalho em professores destacam transtornos mentais, dores no corpo e rouquidão ${ }^{7}$ associados a condições ambientais e organizacionais inapropriadas como salas inadequadas, trabalho repetitivo, exposição ao pó de giz, ambiente estressante, ritmo acelerado de trabalho, desempenho das atividades sem materiais e equipamentos adequados e posição corporal incômoda ${ }^{8}$. Além de violência, pouca margem de autonomia, de criatividade e tempo para o preparo das aulas ${ }^{9}$.

A prevalência de alterações vocais em professores é maior do que na população em geral e a disfonia ocorre de forma diversificada, sendo a forma intermitente a mais preponderante ${ }^{10,11}$.

Em relação aos sintomas vocais, eles podem ser classificados como auditivos, quando é possível ouvir as alterações nas qualidades da voz, como rouquidão, variações do pitch, variações da loudness, falhas na voz, voz fraca e afonia; e sensitivos ou proprioceptivos, que se referem às sensações que o locutor experimenta quando produz a voz, tais como cansaço ao falar, pigarro, dor, ardor e secura, sensação de bola, picada, areia na garganta, entre outras ${ }^{12}$.

Entre os sintomas auditivos, a rouquidão tem sido uma das mais citadas pelos autores decorrente do uso abusivo da voz, e a garganta seca, o cansaço ao falar e o pigarro entre os sintomas proprioceptivos mais referidos motivados pela presença de poeira, ruído no ambiente e baixa hidratação ${ }^{13-16}$. Apesar de tudo isso, é pequena a porcentagem de professores que se afastam da sala de aula devido a problemas vocais ${ }^{17}$.

As pesquisas em voz do professores, além da averiguação de condições insalubres de trabalho e que podem deteriorar a voz docente, tem dado atenção à avaliação das vozes desses profissionais tanto aquela auto-referida pelos sujeitos da pesquisa como a avaliação técnica realizada por profissional fonoaudiólogo. A percepção da qualidade vocal é um parâmetro subjetivo, baseia-se em comparações com outras vozes ou com impressões prévias do ouvinte sobre a mesma voz, além disso, envolvem vários fatores como: características de personalidade, fatores psicológicos e experiência com análise de vozes ${ }^{5,18}$.

Um estudo pesquisou, entre outros aspectos, a percepção acerca de alterações vocais, em 93 educadoras, além da análise perceptivo-auditiva fonoaudiológica de suas vozes e constatou associação estatística entre elas e concluiu que a percepção apropriada das educadoras para seus problemas de voz se constitui em uma estratégia importante nas pesquisas com estes profissionais da voz ${ }^{19}$.

No contexto da discussão sobre a importância de se respeitar à avaliação do professor sobre sua própria voz, assim como a avaliação fonoaudiológica e a busca de estabelecer relações entre elas, o objetivo desse trabalho é tipificar os sintomas vocais relacionados à voz e sua produção auto referidos por professores, cujas vozes foram identificadas como saudáveis na avaliação fonoaudiológica.

\section{MÉTODOS}

Trata-se de pesquisa de corte transversal e caráter quantitativo realizada com 36 professores da rede municipal de ensino da cidade de Salto-SP, com idade mínima de 25 anos, máxima de 57 anos e média de 37 anos, sexo feminino (100\%), predominantemente solteiras $(75 \%)$, escolaridade superior completa $(83,33 \%)$ e tempo médio de docência de 12 anos.

Os professores foram selecionados de uma amostra maior de 165 docentes da rede municipal de ensino de uma cidade do interior do estado de São Paulo que responderam ao questionário proposto por Ferreira et al ${ }^{20}$. Em seguida, foi procedida a gravação digital das vozes dos docentes que constava de emissão de vogais sustentadas e meses do ano, respeitando-se os critérios técnicocientíficos requeridos para esse tipo de coleta de dados. As vozes foram organizadas em CD-ROM e submetidas à avaliação perceptivo-auditiva por três juízas fonoaudiólogas, especialistas em voz, que preencheram um protocolo elaborado especialmente para o presente estudo, no qual assinalavam a ausência ou presença de alteração vocal e nessa última, classificavam a qualidade de voz segundo a proposta de Behlau et al ${ }^{21}$. A avaliação fonoaudiológica final levou em conta o grau de concordância interjuízas e considerada padrão ouro. Em seguida, compararam-se os dados do questionário, em especial a pergunta 49, na qual o professor menciona se tem ou não alteração vocal, com a avaliação das juízas. Desta comparação, se formou um grupo de 36 professores que mencionaram apresentar alteração vocal, dado não confirmado na avaliação fonoaudiológica.

Foram critérios de inclusão nesse estudo: ter referido possuir alteração de voz no instrumento anteriormente mencionado e ter tido sua voz classificada como adaptada na avaliação fonoaudiológica. Foram critérios de exclusão: ter referido presença ou ausência de alteração vocal e ter recebido avaliação fonoaudiológica de voz não adaptada.

Após a seleção dos 36 sujeitos, os dados do questionário relativos aos aspectos sócios

Rev. CEFAC, São Paulo 
demográficos, às condições de trabalho e à saúde foram analisados para caracterizar os professores como grupo e contextualizar o ambiente de trabaIho. Esse pano de fundo foi necessário para a análise e compreensão dos aspectos específicos de voz.

Todos os docentes receberam informações sobre o objetivo e a metodologia do estudo, e concordaram em participar assinando o termo de consentimento. $O$ projeto faz parte de um projeto maior e foi aprovado pelo Comitê de Ética em Pesquisa da PUC-Campinas em 29/10/2007 sob ํo 689/07

Os dados foram organizados em tabelas, comparados e os sintomas vocais tipificados. Não houve possibilidade de aplicação de testes estatísticos comparativos, pois os sujeitos estão organizados em um grupo único, com características de inclusão específicas. Empregou-se somente a análise descritiva dos dados e seus resultados estão apresentados em valores numéricos e percentuais.

\section{RESULTADOS}

O tempo de percepção da alteração vocal pelos professores ficou assim distribuído: 11 (30,56\%) assinalaram mais de quatro anos, nove $(25 \%)$ de 6 meses a 1 ano, sete $(19,44 \%)$ de 0 a 6 meses, cinco $(13,89 \%)$ de 2 a 4 anos e, finalmente, quatro $(11,11 \%)$ de 1 a 2 anos. A forma de início da alteração de voz variou, sendo que $29(80,56 \%)$ docentes indicaram como intermitente e sete $(19,44)$ como progressiva.

Quanto à etiologia da alteração vocal, o uso intensivo da voz foi indicado por $34(94,44 \%)$, estresse por $22(61,11 \%)$, alergia por $20(55,56 \%)$, infecção respiratória, exposição ao frio e exposição ao barulho, cada qual com 14 (38,89\%) professores, gripe constante por sete $(19,44 \%)$ e sem razão aparente por dois $(5,56 \%)$ docentes.

$\mathrm{Na}$ auto-avaliação da sua alteração vocal, 24 $(66,67 \%)$, professores indicaram como sendo moderada, sete $(19,44 \%)$ como leve, três $(8,33 \%)$ como severa e dois $(5,56 \%)$ como grave. Quanto à evolução da alteração vocal, 19 (52,78\%) professores assinalaram com picos de melhora e piora, $10(27,78 \%)$ como estável, cinco $(13,89 \%)$ como tem piorado e dois $(5,56 \%)$ mencionaram que teria melhorado.

Quanto ao número de sintomas, obteve-se que $32(88,89 \%)$ professores referiram três ou mais, sendo que a média mais freqüente foi sete deles, mencionados por sete $(19,44 \%)$ professores.

Para contextualizar os locais onde os sujeitos da pesquisa trabalham e os riscos ocupacionais neles presentes, observou-se que a poeira foi referida por $33(91,67 \%)$ docentes; ambiente ruidoso por
27(75\%); presença de fumaça por 17 (47,22\%); e utilização de produtos químicos irritativos na limpeza por $13(36,11 \%)$. No que diz respeito às condições organizacionais do trabalho, 32 (88,88\%) professores indicaram que não tem tempo para desenvolver as atividades na escola, $28(77,78 \%)$ que o ritmo de trabalho é moderado, e 12 (33,33\%) indicaram fiscalização constante do seu desempenho. No momento da pesquisa, nove $(25 \%)$ docentes trabalhavam em uma escola e 27 (75\%) em duas ou mais. A média de alunos por classe é de 25 alunos, com mínimo de 11 e máximo de 40.

No aspecto de saúde, as queixas foram diversificadas e $29(80,56 \%)$ docentes referiram ansiedade, $19(52,78 \%)$ dores no corpo, 18 (50\%) ganho de peso e dores de cabeça, $15(41,67 \%)$ problemas de coluna, 14 (38,89\%) dores no corpo, 12 (33,33\%) resfriados frequentes, $11(30,56 \%)$ doenças das vias respiratórias, $10(27,78 \%)$ depressão, sete $(19,44 \%)$ azia, cinco (13,89\%) gastrite, três (8,33\%) perda de peso e os problemas de desmaios, tremor, úlcera, doenças crônicas e pânico foram indicados, cada um, por dois $(5,56 \%)$ dos professores.

\section{DISCUSSÃO}

A percepção de professores sobre suas próprias vozes, especificamente quanto à identificação de alterações nelas presentes, tem sido um tema recorrente nas pesquisas fonoaudiológicas. Usualmente, a conclusão dos estudos destaca que esta autopercepção ocorre apenas quando a deterioração das qualidades da voz se encontra em grau severo ${ }^{22}$.

De modo geral, no cotejamento da avaliação vocal realizada por professores e fonoaudiólogos, esta última identifica alguma alteração vocal antes que o docente o faça, o que leva a supor que a percepção de professores nem sempre é apropriada. Entretanto, outras pesquisas podem revelar novas dimensões dessa questão.

No presente estudo, a síntese dos dados sobre a alteração vocal auto-referida pelos docentes mostra tendências, ou seja, o problema é percebido há mais de 4 anos $(30,56 \%)$, com início referenciado como do tipo vai e volta $(80,56 \%)$, consequente ao uso intensivo da voz $(94,44 \%)$, ao estresse $(61,11 \%)$ e a alergia (55,56\%), variando como moderado (66,67\%), e com evolução apresentando picos de melhora e piora $(52,78 \%)$. O tempo de percepção da alteração vocal é longo e, portanto, a alteração antiga e que nenhuma providência efetiva foi tomada para mitigá-la, o que mostra coerência com os resultados de outros estudos 19,20,23. A caracterização do início da alteração vocal como do tipo vai e volta e evolução com picos de melhora e 
piora mostram-se coerentes, e parecem indicar um problema de caráter intermitente e sazonal, que se torna mais presente ou se acentua em condições climáticas específicas, por exemplo, as baixas temperaturas mais agressivas à voz aliadas a aglomeração de pessoas em locais fechados propiciando a disseminação de infecções de vias aéreas, situação típica do ambiente escolar ${ }^{4}$. Esta característica da alteração vocal pode justificar a valoração de moderada atribuída por grande parte dos professores à sua alteração vocal. A origem da alteração vocal é associada pelos docentes ao uso intensivo da voz, estresse e alergia, fatores possivelmente decorrentes das condições nem sempre apropriadas de trabalho, em especial a presença de ruído ${ }^{2,17,24}$ poeira que pode desencadear processos alérgicos ${ }^{2,10,25}$ estresse, decorrente do excesso de trabalho e alunos ${ }^{23,26}$.

Especificamente em relação ao número de sintomas referidos pelos docentes, ele se mostrou variado, com maior frequência de sete $(19,44 \%)$ e seis sintomas (13,89\%). Considerando-se que a partir de três sintomas há indicação de fadiga vocal ${ }^{27}$ os professores desse estudo, mesmo não sendo identificados pela avaliação fonoaudiológica a presença de alteração vocal, exibem uma sintomatologia auditiva e proprioceptiva variada e frequente, o que requer atenção e cuidado.

Os sintomas auditivos (Figura 1) foram diversificados, com predomínio em frequência da rouquidão $(69,44 \%)$ e voz grossa $(41,66 \%)$. A rouquidão é um sintoma muito frequente em pesquisas nacionais e internacionais que estudam a voz do professor ${ }^{2,14,16,17,28} \mathrm{e}$ indica agressão à laringe e pregas vocais decorrente, usualmente, do uso abusivo da voz. Da mesma forma, a voz grossa $(41,66 \%)$ pode secundária ao edema de pregas vocais, decorrente de agressão por fonotrauma e/ou poeira que resulta em perda temporária das frequências agudas, tudo isso culminando com a voz avaliada como fraca $(38,88 \%)$ pelos professores, sugestiva de desgaste vocal ou fonastenia ${ }^{17}$.

Já em relação aos sintomas do tipo proprioceptivo (Figura 2), os mais referidos pelos docentes foram o cansaço ao falar $(69,44 \%)$, o pigarro $(63,88 \%)$ e garganta seca $(61,11 \%)$. Esses sintomas guardam relação entre si, inicialmente porque fadiga vocal é frequente entre os docentes ${ }^{29}$ e pode ser decorrente de excesso de força para falar ${ }^{30}$, além de falta de hidratação o que gera garganta seca e o pigarro como forma de reação ao esforço vocal ${ }^{2}$. Esses dados são coerentes com a indicação dos professores de que a origem de suas alterações vocais encontra-se no uso intensivo da voz e podem mostrar consonância com as adversidades mencionadas no ambiente e organização do trabalho.

A comparação dos tipos de sintomas auto referidos pelos sujeitos desse estudo (Figura 3) mostra que os proprioceptivos $(63,26 \%)$ são mais frequentes que os auditivos (36,73\%), podendo indicar que é o professor aquele que mais percebe seu esforço vocal, pois ocorrem sensações e incômodos na garganta invisíveis para o interlocutor. Isto mostra que mesmo sem alterações vocais importantes, o processo de adoecimento vocal já está em curso e, se não interceptado esse processo, logo as qualidades da voz serão prejudicadas. Além disso, a relação entre os sintomas proprioceptivos e auditivos por professor (Figura 4) indica que os proprioceptivos estão em maior número que os auditivos em 25 sujeitos $(69,44 \%)$.

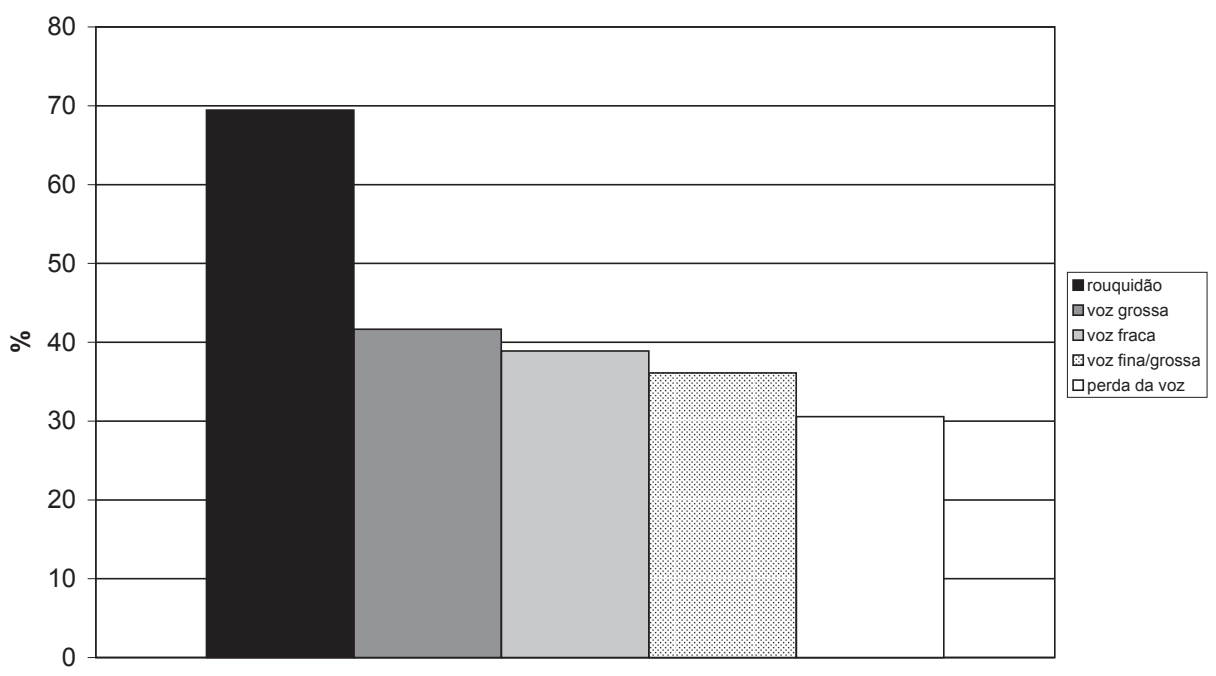

Figura 1 - Sintomas auditivos mais frequentes auto referidos pelos docentes 


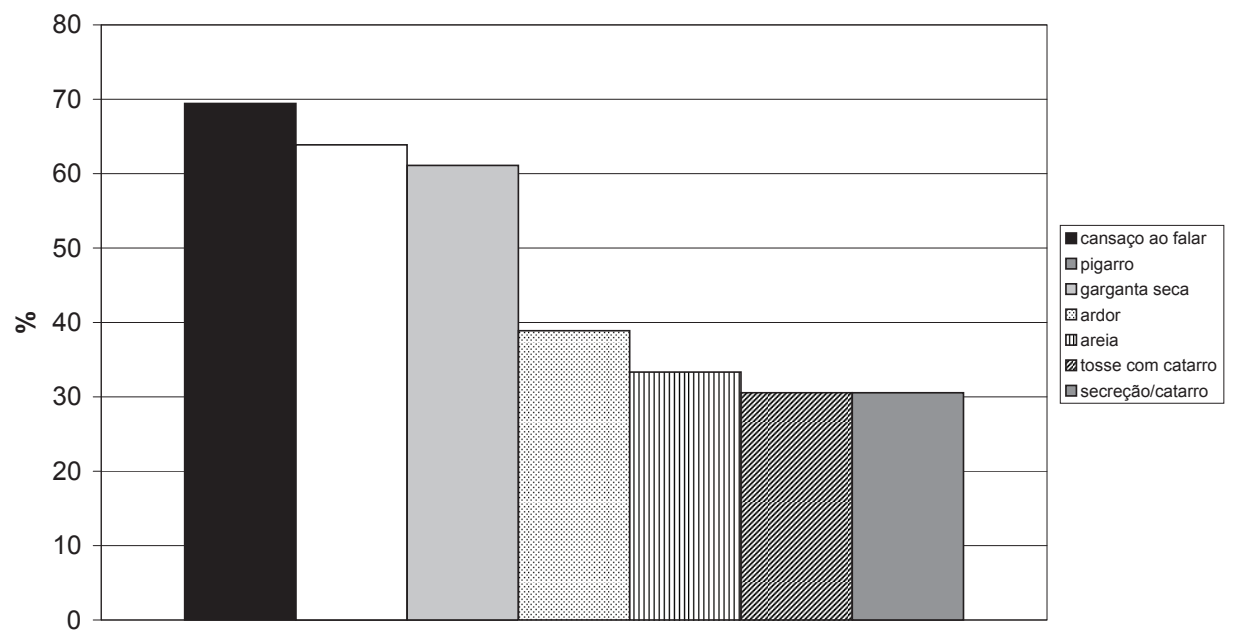

Figura 2 - Sintomas proprioceptivos ligados à voz e à garganta

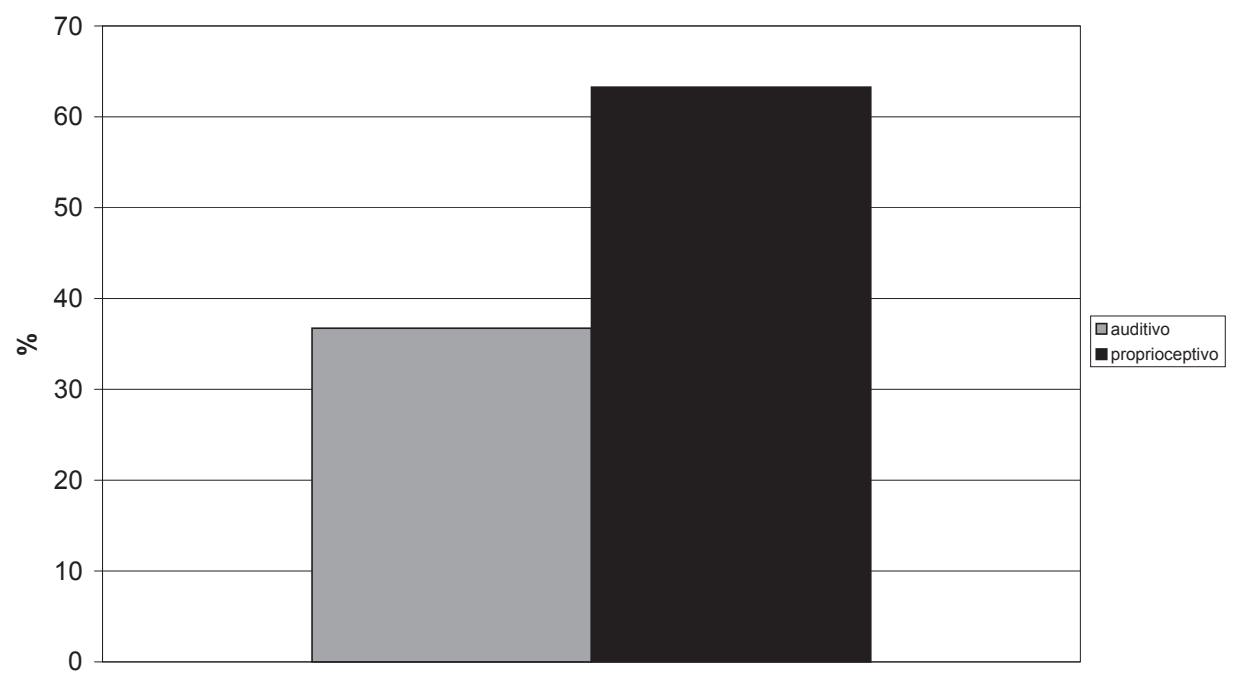

Figura 3 - Comparação entre a frequência dos diferentes tipos de sintomas mencionados pelos docentes

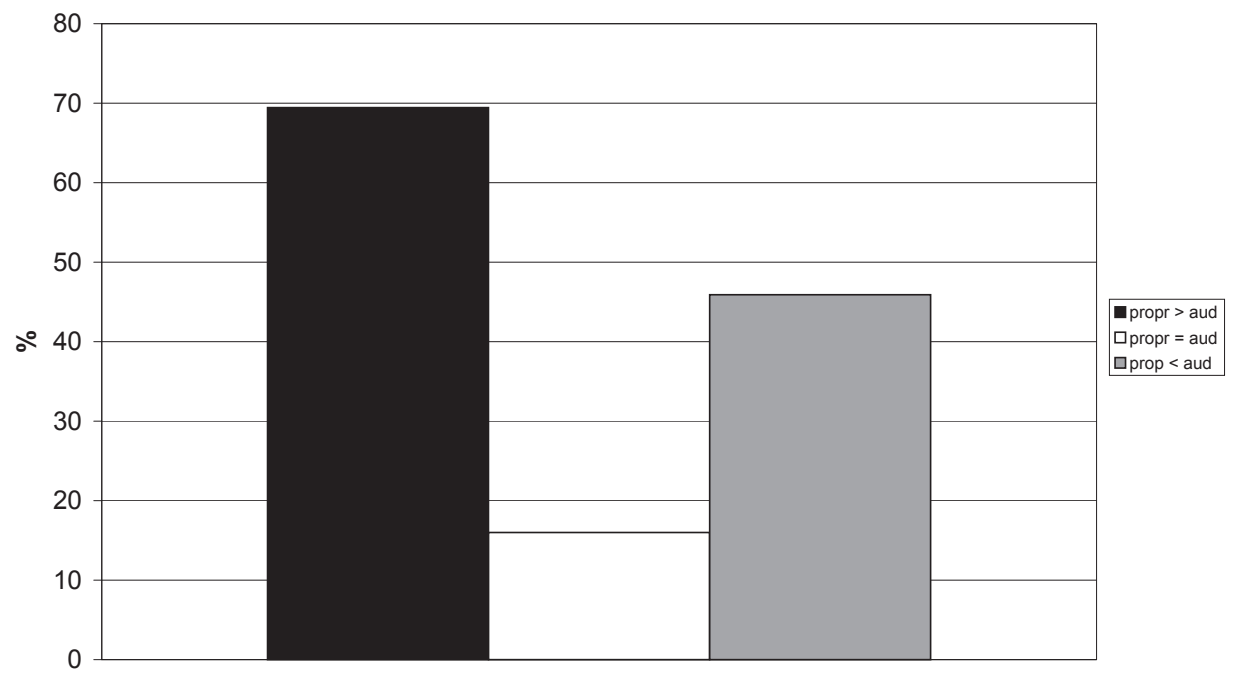

Figura 4 - Relação entre a quantidade de sintomas proprioceptivos e auditivos 
Esses dados podem justificar porque os professores mencionaram a alteração vocal, a qual não foi captada pelos fonoaudiólogos. $\mathrm{Na}$ avaliação realizada pelas juízas, elas não contavam com nenhum dado sobre os professores exceto sua emissão de vogais e meses do ano. Embora a rouquidão tenha sido o sintoma auditivo mais referido pelos docentes, possivelmente seu grau não tenha sido suficientemente grave para ser identificado, em especial quando a gravação foi realizada, considerando-se que os professores assinalaram que a alteração vocal é do tipo vai e volta. Outra possibilidade a se considerar é que as amostras de voz, embora valiosas, possam ter sido muito curtas para se detectar o processo de disfonia. Quando a avaliação é feita na situação face a face, o fonoaudiólogo dispõe de múltiplas informações e pistas que, ao serem articuladas, provêem maior possibilidade de diagnóstico vocal.

Os resultados obtidos contribuem para desmistificar o fato de que os professores não percebem suas alterações vocais ou que apenas as mencionem quando elas se encontram em um estado de gravidade acentuado ${ }^{4,22}$. Isto não ocorreu nesse estudo, podendo ser uma oportunidade para que o fonoaudiólogo observe que o professor como dono da voz é sensível às suas modificações e que a consequente procura por ajuda fonoaudiológica pode estar vinculada a outras questões como falta de acesso a esse tipo de serviço, seja pela escassez de fonoaudiólogos nos serviços públicos de saúde, seja pela falta de disponibilidade de horários que acolham os docentes que atuam em jornada dupla.

A consideração dessas questões abre espaço para mais pesquisas e articulações entre os docentes e os fonoaudiólogos, cuja parceria tem a chance de trazer mudanças político-administrativas que atendam às necessidades de saúde e voz do professor.

\section{CONCLUSÃO}

O presente estudo mostrou que os professores avaliados trabalham em condições de trabalho desfavoráveis, as quais podem colaborar para o uso intensivo e abusivo da voz.

A tipificação dos sintomas relacionados à voz e sua produção auto referidos pelos docentes mostrou que houve prevalência de sintomas proprioceptivos $(63,26 \%)$ em comparação com os auditivos $(36,73 \%)$. Esse fato pode ter colaborado para que a avaliação fonoaudiológica não identificasse a presença de alterações, pois as juízas só tiveram acesso a uma amostra da voz dos docentes.

Considerando-se a legitimidade da avaliação vocal tanto das docentes quanto das fonoaudiólogas, esse estudo indica que a combinação dos dois tipos contribui para uma visão mais integral da voz do professor, pois conjuga a visão de vários segmentos e saberes e mostra-se como uma estratégia interessante na compreensão das relações entre saúde e voz na docência.

\section{AGRADECIMENTOS}

À Secretaria de Educação da Estância Turística de Salto que acolheu o projeto de pesquisa e à PUC-Campinas pelo apoio financeiro e logístico para a realização do estudo. 


\begin{abstract}
Purpose: to typify the symptoms related to voice and its production self-referred by teachers, whose voices were identified as healthful in the Speech-Language Pathology evaluation. Methods: 36 teachers took part, with mean age of 37 years, singles (75\%) and college degree $(83.33 \%)$, from the county school system of a city from the countryside of São Paulo. The teachers answered a questionnaire and referred the alteration; their voices were recorded and submitted to the perceptual Speech-Language Pathology evaluation and afterwards, both types of evaluation were compared. The socio-demographic characterization proceeded, environmental and organizational conditions of the teacher's work and their symptoms typified and submitted to a statistical analysis. Results: the presence of dust $(91.67 \%)$, noise $(75 \%)$, excess of work $(88.88 \%)$, lack of time to develop the activities at school (88.88\%) and constant performance monitoring (33.33\%) were noted. As for the voice, the alteration has been noted for more than four years (30.56\%), secondary to its intensive use $(94.44 \%)$ and to stress $(61.11 \%)$, classified as moderate $(66.67 \%)$, being prominent like auditory symptoms, hoarseness and tiredness of speaking, both with $69.44 \%$ and classified as proprioceptive symptoms, throat clearing $(63.88 \%)$ and dry throat $(61.11 \%)$. The comparison among the symptoms shows that the proprioceptive ones (63.26\%) were more mentioned than the auditory ones (36.73\%). Conclusion: the teachers work in health and voice adverse environment; the prevalence of proprioceptive symptoms was greater than the auditory ones, a fact that may have interfered in the perceptual evaluation of their voices by the Speech-Language Pathologists who just counted on the auditory clue.
\end{abstract}

KEYWORDS: Occupational Health; Voice; Voice Disorders; Faculty; Voice Quality

\section{REFERÊNCIAS}

1. Rogerson J, Dodd B. Is there an effect of dysphonic teachers' voices on children's processing of spoken language? J Voice. 2005; 19(1):47-60.

2. Smolander S, Huttunen K. Voice problems experienced by Finnish comprehensive school teachers and realization of occupational health care. Logoped Phoniatr Vocol. 2006; 31(4):166-71.

3. São Paulo. Centro de Referência em Saúde do Trabalhador. Coordenadoria de Controle de Doenças (CEREST/CCD).Distúrbios de voz relacionados ao trabalho. Bol Epidemiol Paul. 2006; 3(26):16-22.

4. Yiu EM. Impact and prevention of voice problems in the teaching profession: embracing the consumers' view. J Voice. 2002; 16(2):215-28.

5. Duffy OM, Hazlett DE. The impact of preventive voice care programs for training teachers: a longitudinal study. J Voice. 2004; 18(1):63-70.

6. Krischke S, Weigelt S, Hoppe U, Köllner V, Klotz $M$, Eysholdt $U$, et al. Quality of life in dysphonic patients. J Voice. 2005; 19(1):132-7.

7. Delcor NS, Araújo TM, Reis EJFB, Porto LA, Carvalho FM, Silva MO, et al. Condições de trabalho e saúde dos professores da rede particular de ensino de Vitória da Conquista, Bahia, Brasil. Cad Saúde Pública. 2004; 20(1):187-96.
8. Silvany Neto AM, Araújo TM, Dultra FRD, Azi GR, Alves RL, Kavalkievick C, et al. Condições de trabalho e saúde de professores da rede particular de ensino de Salvador, BA. Rev. Baiana Saúde Pública. 2000; 24(1/2):42-56.

9. Gasparini SM, Barreto SM, Assunção AA. Prevalência de transtornos mentais comuns em professores da rede municipal de Belo Horizonte, Minas Gerais, Brasil. Cad Saúde Pública. 2006; 22(12):2679-91.

10. Fuess VLR, Lorenz MC. Disfonia em professores do ensino municipal: prevalência e fatores de risco. Rev Bras Otorrinolaringol. 2003; 69(6):807-12.

11. Munier C, Kinsella R. The prevalence and impact of voice problems in primary school teachers. Occup Med. 2008; 58(1):74-6.

12. Cooper M. Modernas técnicas de reabilitación vocal. Buenos Aires: Panamericana; 1973.

13. Jardim R. Voz, trabalho docente e qualidade de vida [dissertação]. Belo Horizonte (MG): Universidade Federal de Minas Gerais; 2006.

14. Preciado J, Pérez C, Calzada M, Preciado P. Frequencia y factores de riesgo de los trastornos de la voz en el personal docente de La Rioja. Estudio transversal de 527 docentes: cuestionario, examen de la función vocal, análisis acústico y vídeolaringoestroboscopia. Acta Otorrinolaringol Esp. 2005; 56(4):161-70. 
15. Simões M, Latorre MRDO. Alteração vocal em professores: uma revisão. J Bras Fonoaudiol. 2002; 3(11):127-34.

16. Sliwinska-Kowalska M, Niebudek-Bogusz E, Fiszer M, Los-Spychalska T, Kotylo P, SznurowskaPrzygocka B, et al. The prevalence and risk factors for occupational voice disorders in teachers. Folia Phoniatr Logop. 2006; 58(2):85-101.

17. Tavares EL, Martins RH. Vocal evaluation in teachers with or without symptoms. J Voice. 2007; 21(4):407-14.

18. Bele IV. Reliability in perceptual analysis of voice quality. J Voice. 2005; 19(4):555-73.

19. Simões $M$, Latorre MRDO. Prevalência de alteração vocal em educadoras e sua relação com a auto-percepção. Rev Saúde Pública. 2006; 40(6):1013-8.

20. Ferreira LP, Giannini SPP, Figueira S, Silva EE, Karmann DF, Souza TMT. Condições de produção vocal de professores da Prefeitura do Município de São Paulo. Dist Comun. 2003; 14(2):275-307.

21. Behlau M, Damazio G, Feijó D, Pontes P. Avaliação de voz. In: Behlau M. Voz. O livro do Especialista. Vol. 1. Rio de Janeiro: Revinter; 2001. p. $85-180$.

22. Penteado RZ, Bicudo-Perreira IMT. Avaliação do impacto da voz na qualidade de vida de professores. Rev Soc Bras Fonoaudiol. 2003; 8(2):19-28.

23. Thomas G, Kooijman PG, Cremers CW, de Jong FI. A comparative study of voice complains and risk factors for voice complaints in female student teachers and practicing teachers early in their career. Eur Arch Otorhinolaryngol. 2006; 263(4):370-80.

24. Petter V, Oliveira PAB, Fischer PD. Relación entre disfonía referida y potenciales factores de riesgo en el trabajo de profesores de la enseñanza fundamental, Porto Alegre - RS. Salud de los Trabajadores. 2006; 14(2):5-12.

25. Ortiz E, Alves Lima E, Costa E. Saúde vocal de professores da rede municipal de ensino de cidade do interior de São Paulo. Rev Bras Med Trab. 2004; 2(4):263-6.

26. Simberg S, Sala E, Vehmas K, Laine A. Changes in the prevalence of vocal symptoms among teachers during a twelve-year period. J Voice. 2005; 19(1):95-102.

27. Sapir S, Keidar A, Mathers-Schmidt B. Vocal attrition in teachers: survey findings. Eur $\mathrm{J}$ Disord Commun. 1993; 28(2):177-85.

28. Hamdan AL, Sibai AM, Srour ZM, Sabra OA, Deeb RA. Voice disorders in teachers: the role of family physicians. Saudi Med J. 2007; 28(3):422-8.

29. Gotaas C, Starr CD. Vocal fatigue among teachers. Folia Phoniatr. 1993; 45(3):120-9.

30. Chang A, Karnell MP. Perceived phonatory effort and phonation threshold pressure across a prolonged voice loading task: a study of vocal fatigue. J Voice. 2004; 18(4):453-66.

RECEBIDO EM: 01/08/2009

ACEITO EM: 17/12/2009

Endereço para correspondência:

Emilse Aparecida Merlin Servilha

Av. John Boyd Dunlop, s/no

Campinas - SP

CEP: 13060-904

E-mail: emilsemerlin@uol.com.br 